\title{
Towards a Standard Approach to Assess Tibial Bone Loss Following Total Knee Arthroplasty
}

\author{
Thomas Anijs $^{1}\left[\right.$ D Ilse Kouwert ${ }^{1} \cdot$ Nico Verdonschot $^{1,2} \cdot$ Dennis Janssen $^{1}$
}

Accepted: 13 July 2021 / Published online: 22 July 2021

(c) The Author(s) 2021

\begin{abstract}
Long-term implant failure in the form of aseptic loosening and periprosthetic fracture is the most common cause of revision procedures in total knee arthroplasty (TKA). While early loosening can often be attributed to failure of primary fixation, late implant failure could be associated with loss of fixation secondary to bone resorption, as a result of stress shielding in the proximal tibia. This current review study was performed to identify the clinical effects of different implant-, patient-, and surgery-related biomechanical factors on TKA-related tibial bone loss in clinical reality. Implant-related factors considered were the fixation method, and the implant type, geometry, and stiffness. In terms of patient characteristics, the effects of age, sex, knee alignment, bone density, body weight, and activity level were analyzed. The clinical literature on these topics mostly concerned longitudinal radiographic studies investigating the effect of a single factor on changes in the proximal tibia over time using bone densitometry. Implant stiffness, implant geometry and knee alignment were the only factors consistently found to affect regional bone density changes over time. Each clinical study used its own specific study design, with different definitions used for the baseline density, time points of baseline and follow-up measurements, and regions of interest. Due to the differences in study design, direct comparison between the clinical impact of different biomechanical factors was not possible. Based on the findings over the densitometry studies, a standardized guideline was proposed to allow reliable comparison between consistently reported outcome of future radiographic TKA studies.
\end{abstract}

Keywords Total knee arthroplasty $\cdot$ Bone remodeling $\cdot$ Proximal tibia $\cdot$ DEXA $\cdot$ Bone mineral density $\cdot$ Densitometry

\section{Introduction}

Total knee arthroplasty (TKA) is one of the most successful surgical interventions, but the number of primary TKA failures is increasing as a result of the aging population and the acceptance of TKA in younger patients [1]. Two common causes of long-term tibial implant failure are aseptic loosening and periprosthetic fracture [1,2], which can be linked to stress shielding-related osteolysis observed around the implant [3]. Due to an alteration of stress distribution in the bone after TKA, by the introduction of a homogenous

Thomas Anijs

Thomas.Anijs@radboudumc.nl

1 Orthopaedic Research Laboratory, Radboud Institute for Health Sciences, Radboud University Medical Center, Nijmegen, The Netherlands

2 Laboratory for Biomechanical Engineering, Faculty of Engineering Technology, University of Twente, Enschede, The Netherlands implant and change in physiological loading, adaptive bone remodeling will take place over time following Wolff's law [4], which typically leads to periprosthetic bone resorption [5]. Many longitudinal clinical studies have been conducted on postoperative bone changes, by measuring bone density in the proximal tibia over time. The most commonly used densitometry method is dual-energy X-ray absorptiometry (DEXA) [6], but also, quantitative computed tomography (QCT) [7] and digital radiograph densitometry (DRD) [8] have been used, to enable measurements in volumetric regions and from standard radiographs, respectively. The goal of most studies was to establish the effect of a single factor in TKA on subsequent regional density changes as a result of bone remodeling.

A narrative literature review was conducted in this study, investigating the impact of different TKA variations on tibial density and giving an overview of the variety in study design, since it is currently unclear what the relative effects of different implant- and patient-related variations are. Following the review outcome, a general guideline was 
proposed for the study design of future studies on periprosthetic bone density changes, since the vast differences in designs of current studies prevent direct comparison between reported results of different studies. A general study design will improve understanding of the relative impact of different TKA factors and effectiveness in reducing postoperative bone loss, to prevent related late tibial implant failure.

\section{Methods}

In this literature study, longitudinal clinical studies covering the effect of mechanical factors on bone density changes after TKA were reviewed. A distinction was made between factors related to the implant, patient, and surgery. Four categories of implant-related factors were identified: fixation method (cemented and uncemented fixation), implant stiffness (material stiffness and structural baseplate stiffness), implant geometry, and implant type (type of bearing and cruciate ligament retention). Regarding patient and surgery features, the effects of six different factors were investigated: age, sex, knee alignment (change), preoperative bone density, body weight, and activity level. These factors were determined based on variations analyzed in relevant studies, considering potential change of the mechanical stress redistribution in the tibia and subsequent bone response. Based on the different used methods and findings over the studies, conclusions were drawn on the effects of the discussed factors on long-term bone density outcome, and recommendations were provided for future densitometry studies regarding study design and reporting of relevant data.

\section{Literature Findings}

Seventeen different clinical studies were included covering implant-related factors (Table 1), while eleven studies were consulted following the patient- and surgery-related factors (Table 2). Studies covering the effects of multiple factors on bone density changes have been reviewed more than once over this section.

\section{Effects of Implant-Related Factors on Tibial Bone Density Changes}

\section{Fixation Method}

Tibial TKA components can be fixated to the bone using either a cemented fixation or a cementless press-fit fixation. Three clinical studies reported on the effect of fixation method in otherwise similar implants in periprosthetic tibial density changes [9-11] (Table 1). A study by Small et al. [9], based on DRD, found a significant reduction of bone loss around cemented implants relative to uncemented components at 5 years after TKA, but no significant differences at other time intervals up to 10 years. The same study also found that tibial density changes were significantly related to body mass index (BMI) in the cemented group, but not in the uncemented group, although no explanation for this observation was suggested [9]. Abu-Rajab et al. [10] reported no difference between cemented and uncemented fixation in the extent of relative bone mineral density (BMD) difference compared to the unoperated contralateral knee after a minimum postoperative time of 2 years. In a study by $\mathrm{Li}$ and Nilsson, no significant differences in relative density changes between the fixation methods were seen within any follow-up period up to 2 years [11]. Interestingly, implant migration, determined using radiostereometric analysis (RSA), was found to be related to preoperative BMD in the uncemented group up until 6 months after operation, but not in the cemented cohort [12]. In the same patient cohort, no relationship between migration and 2-year BMD changes has been established [13]. Another DEXA study comparing cemented and uncemented implants found reduced bone loss following uncemented implants, but both implant systems also differed distinctively in material stiffness and stem design [14].

Within uncemented implants, a distinction can be made in the type of coating at the bone contact surfaces, which may affect the course of periprosthetic bone remodeling [15, 16] (Table 1). One study reported a significant difference in lateral proximal density between hydroxyapatite-coated and uncoated implants, with higher BMD values found in the uncoated group at 2 years postoperatively [16]. In contrast, another study with similar implants did not find any density differences between different types of coating [15].

In summary, none of the abovementioned studies attributed measured differences in bone resorption solely to the implant fixation method. One study reported a relationship between BMI and bone density change in cemented implants, which was significantly different from uncemented implants only at the 5-year time point [9], providing minimal evidence for the role of fixation method in the course of periprosthetic bone remodeling.

\section{Implant Stiffness}

As periprosthetic bone loss is the result of a reduction in local bone stress, caused by the high stiffness of (metallic) implants compared to the replaced bone tissue, it is hypothesized that this stress shielding is reduced when using implants with a decreased stiffness. One way to achieve this is through the use of implant materials with a lower modulus of elasticity. Geometry may also affect the stress shielding potential of a specific implant, as the structural stiffness can be lowered by decreasing the thickness of the tibial baseplate. 


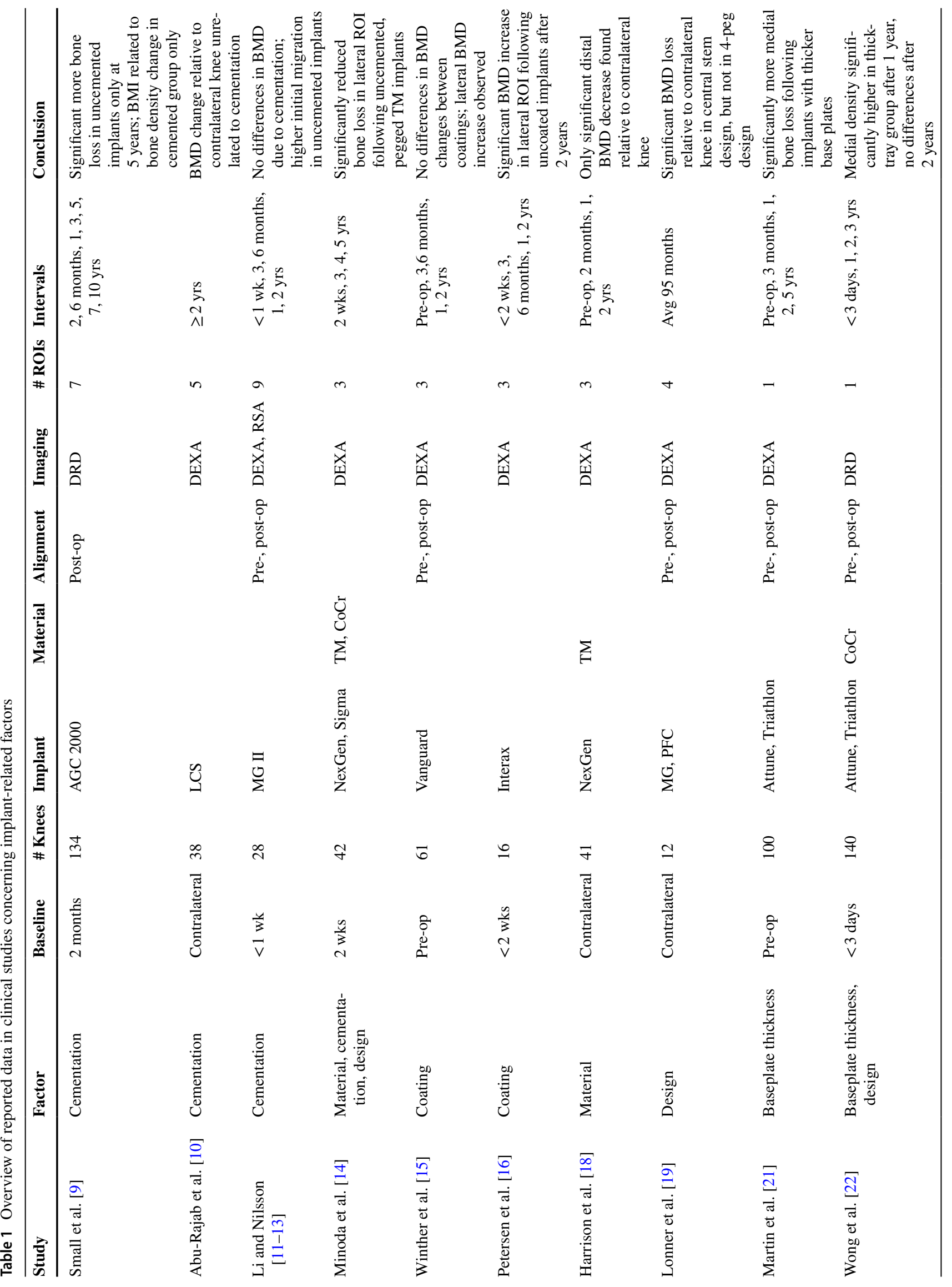




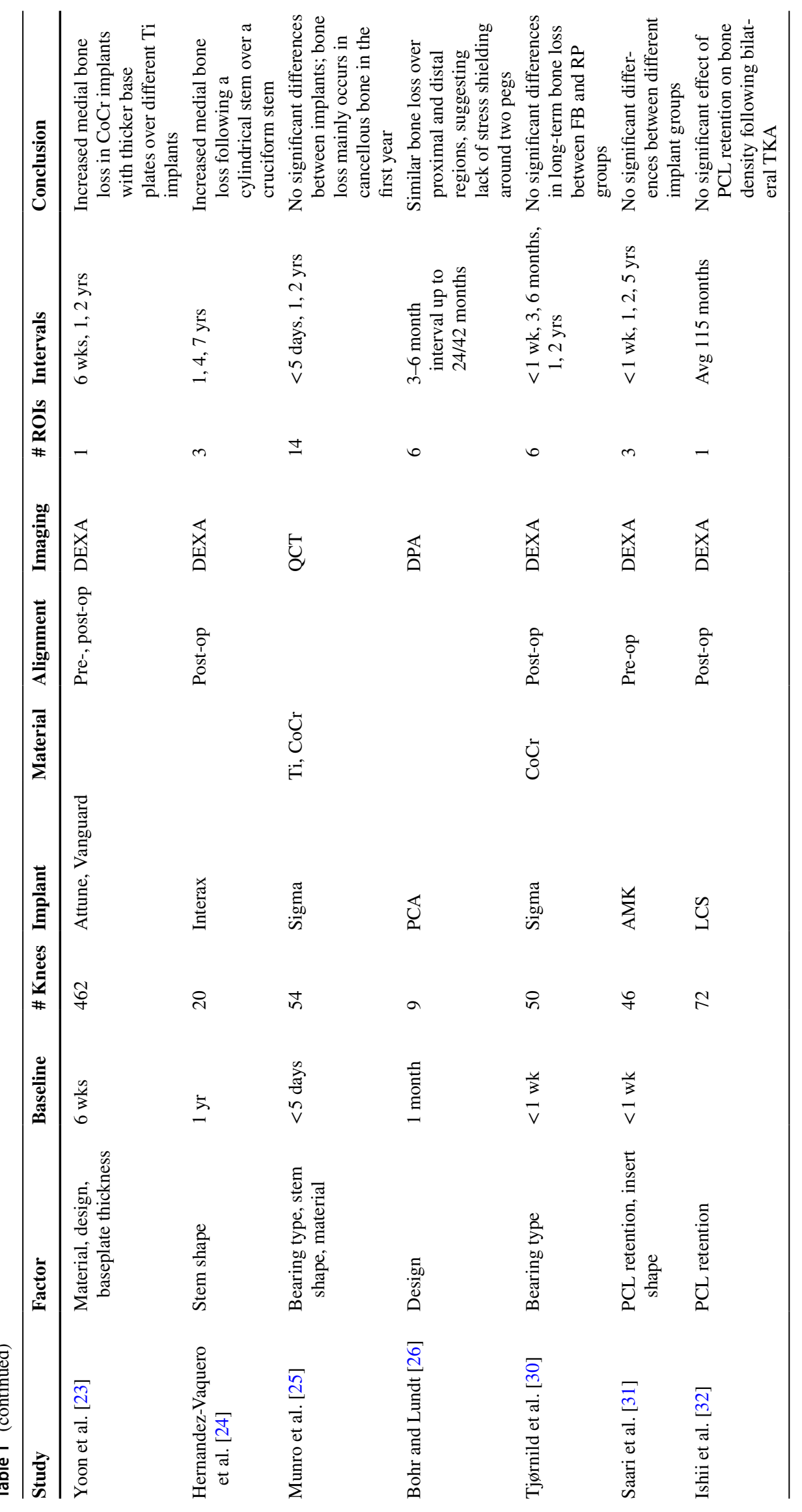




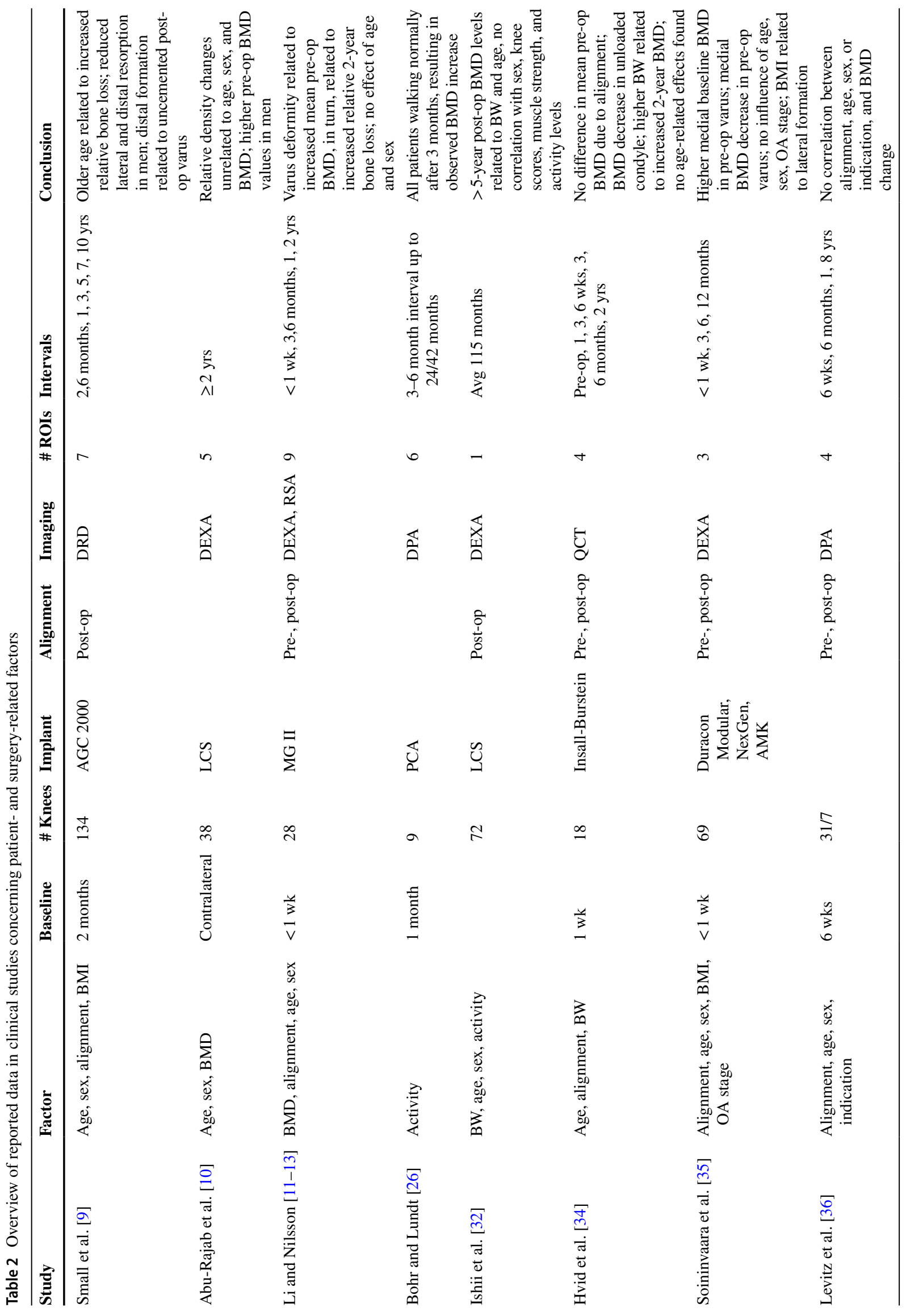




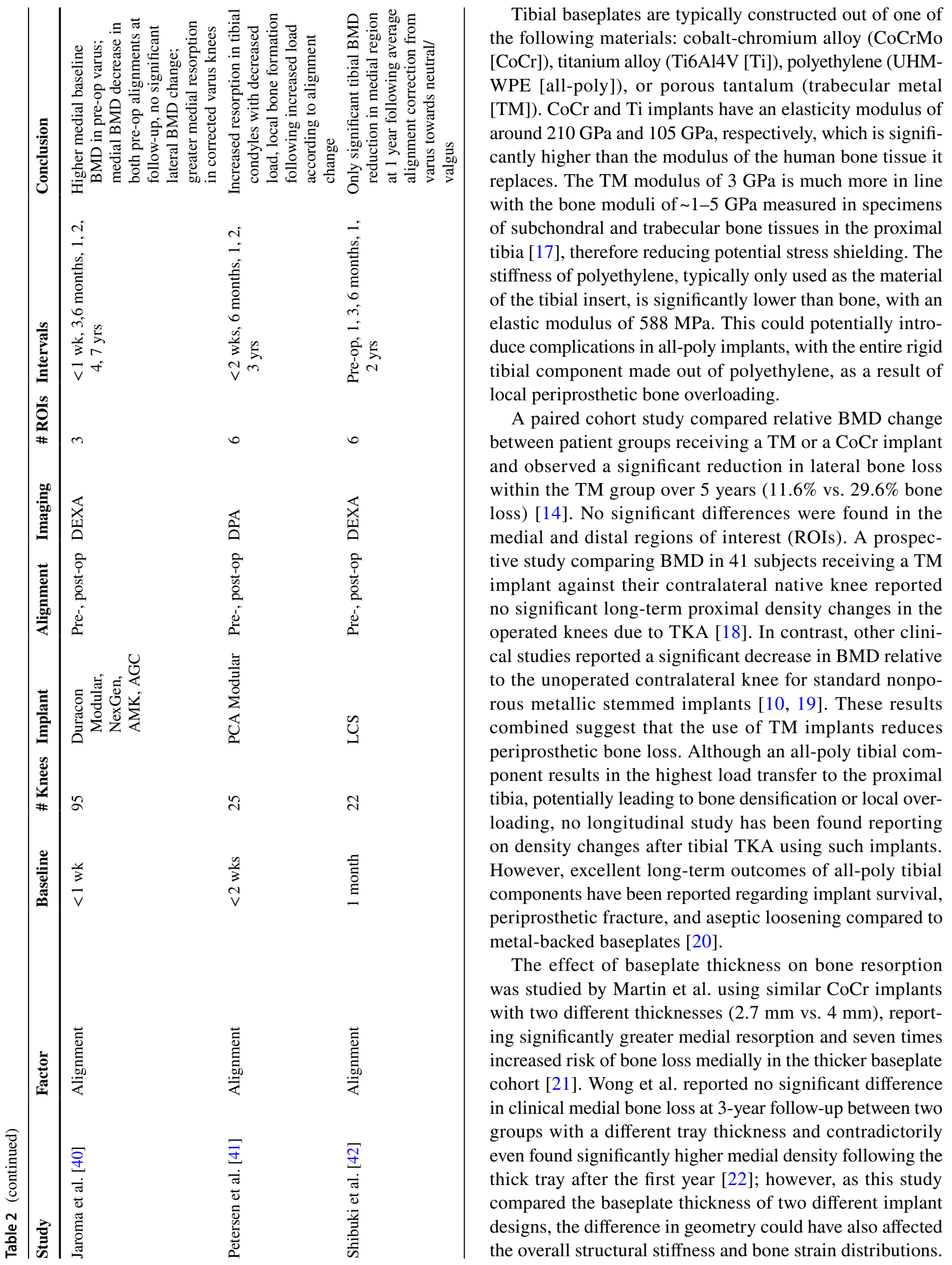


Findings by Yoon et al. endorsed the theory that implants with greater overall stiffness lead to increased bone resorption, by reporting a greater degree of medial bone loss in various $\mathrm{CoCr}$ implants compared to lower modulus $\mathrm{Ti}$ implants with smaller baseplate thicknesses [23].

\section{Implant Geometry}

Besides thickness of the baseplate, there are additional design features that may influence periprosthetic bone density. The shape of the baseplate determines the coverage of the resected tibial bone, and therefore, the transfer of loads from the joint to the bone. There is also a variety of fixation features, which typically include a central stem and/ or smaller pegs at the medial and lateral condyles (Fig. 1). Stem type and shape affect the way strains are transferred through the tibia and, to a lesser extent, influence the structural baseplate stiffness. Several studies have been conducted to study their effect on clinical BMD measurements [14, 19, 24, 25] (Table 1).

The effect of the shape of the central stem was demonstrated in a study where a cylindrical stem showed increased and more concentrated medial bone loss compared to a cruciform-shaped stem, in which BMD decrease was more evenly spread over the proximal tibia, in an otherwise identical design [24]. A different study, comparing cruciform and cylindrical stems with different implant bearings, did not find any evidence for bone density differences up to 2 years [25]. Comparison between an implant with four short fixation pegs, and another cemented implant with a larger cylindrical central stem, found a significant proximal BMD reduction for the single-stem implant compared to the contralateral control, but not for the four-pegged design [19]. Minoda et al. found a similar difference between both distinct stem types [14]. However, multiple factors were varied simultaneously in this study, as the cylindrical single-stem component was also constructed out of a much stiffer material [14]. A single-implant study by Bohr and Lund found a high correlation between BMD of proximal and distal areas of the tibia over follow-up in an uncemented metallic implant with two fixation pegs and suggested that no stress shielding occurs around these smaller pegs [26]. However, a recent register study concluded that a single design of a cemented four-pegged baseplate had a higher risk of aseptic loosening than the corresponding implant using a single central stem [27]. Two single-center studies with a followup greater than 5 years did not find a difference in clinical outcome between both fixation options in two knee systems $[28,29]$.

\section{Implant Bearing Type}

Tibial components can be subdivided in either fixed bearing (FB) or rotating platform (RP) implants. RP components provide an additional rotational degree of freedom at the interface between the polyethylene insert and the tibial baseplate when compared to traditional FB implants. RP implants can therefore theoretically reduce shear stress at the contact area between the femoral component and insert and may affect stress shielding by facilitating a more equal distribution of compressive forces and reducing the axial torque acting on the tibial component.

In addition, a distinction can be made on the type of constraint of the articulating surface, with the most popular types being the cruciate retaining (CR) and posterior stabilized (PS) implant. While, in CR implants, the posterior cruciate ligament (PCL) is (at least partially) responsible for the anteroposterior (AP) stability, these forces are mainly transferred through the post-cam mechanism in PS implants, which, in turn, may affect the stresses in the periprosthetic bone.

Three different joint constraint studies found similar bone density reduction in FB and RP designs of a single implant system, indicating that bone resorption was not related to PCL retention [30-32] (Table 1). Additionally, Saari et al. also varied the shape of the bearing surface (flat vs. concave); the results indicated that the shape of the contact surface also did not affect periprosthetic BMD up to 5 years [31]. Similarly, a QCT-based investigation by Munro et al. did not show differences in BMD loss between
Fig. 1 Schematic impression of different fixation features used in tibial implant designs

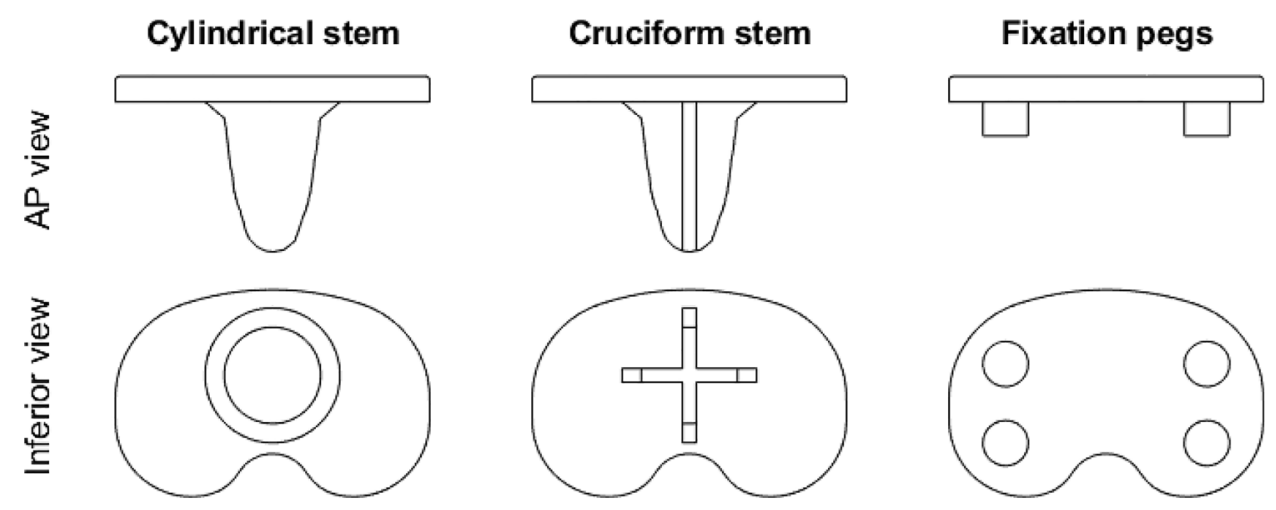


RP and FB implants, although the implants in this particular study also had different stem shapes and were constructed from different nonporous metals ( $\mathrm{FB}$-cruciform, Ti vs. $\mathrm{RP}$ — cylindrical, $\mathrm{CoCr}$ ) [25].

\section{Effects of Patient- and Surgery-Related Factors on Tibial Bone Density Changes}

\section{Age}

Bone density decreases with increasing age in a general non-TKA population, as observed in BMD measurements of three different bone sites across different age groups with a range of 29 to 87 years old [33]. The age of the patient, therefore, affects the initial BMD at the time of surgery, which could influence the subsequent progress of periprosthetic bone remodeling. However, since TKA is generally performed in older patients, the age range of a primary TKA cohort is limited. Several studies have therefore been unable to demonstrate an age-related effect on initial mean proximal tibial BMD, and on bone density changes after TKA [10, 13, 34-36] (Table 2). Conversely, Small et al. did find that higher age at time of surgery was associated with an increase in bone density loss after TKA (in lateral and distal regions) [9]. Similarly, Ishii et al. found a weak negative correlation between age and postoperative BMD formation [32].

\section{Sex}

General age-related bone loss is more pronounced in females, due to postmenopausal-related effects [33]. Consequently, higher baseline BMD levels have been found in male than in female TKA patients, but this did not result in differences in relative BMD changes when compared to the contralateral knee [10]. These findings were in line with several other studies that were unable to demonstrate significant differences in postoperative BMD changes by sex [13, 32 , 35, 36] (Table 2). Conversely, a study by Small et al. found significantly less bone loss in male patients than in female patients in all lateral and distal regions [9], possibly caused by correction of preoperative varus deformity in unreported native knee alignment, which is more common in men than in women [37], leading to a shift in load distribution towards lateral. No studies were found considering menopausal status regarding bone loss after TKA.

\section{Knee Alignment}

In line with Wolff's Law, the mediolateral (ML) bone density distribution in intact tibiae has been found to vary based on native knee alignment [11, 38, 39]. TKA patients with varus preoperative alignment, therefore, have a higher baseline BMD in the medial compartment, while valgus knees typically show greater initial lateral densification $[11,12$, $35,40]$. In terms of overall mean density over the proximal tibia, Hvid et al. and Levitz et al. found no significant difference between preoperative varus and valgus knees [34, 36], while Li and Nilsson found greater general BMD in knees with native varus alignment [11]. Interestingly, this study also found a greater relative bone resorption in native varus knees 2 years after TKA, while subsequent postoperative alignment was not found to be a predictor for relative 24-month BMD change [11].

The same study also investigated the effect of intrasubjective alignment change by making a distinction between compartments based on increase or decrease in load following the alignment difference (e.g., an increase in load was assumed in the lateral compartment when correcting a varus knee to neutral). They found an increase in bone formation underneath the load-increased condyle over the load-decreased side, but only in patients with a low mean baseline BMD over the proximal tibia [11]. A different study, based on dual photon absorptiometry (DPA), demonstrated a similar effect of alignment change, with extensive resorption observed in the compartment with reduced postoperative loading, and a slight but significant increase in density in the compartment with increased load [41]. Several other studies demonstrated a BMD decrease in the load-decreased condyle but did not find a significant density change in the load-increased side based on the change between pre- and postoperative varus angles [34, 35, 42]. Hvid et al. found this effect solely in the lateral region, with no significant decrease of medial density in the preoperative varus group separately after 2 years [34], while other studies only found significant differences in medial decrease related to preoperative varus knees at 1-year follow-up [35, 42]. Jaroma et al. also reported a significant medial decrease of density in valgus preoperative knees, while no significant density changes were found in the lateral ROI, regardless of preoperative alignment [40]. The extent of medial resorption was found to differ significantly between postoperative alignments within the varus preoperative group, with considerably more relative resorption in alignments towards valgus [40]. Densification in the distal region, underneath a central implant stem, was found to be related to postoperative varus alignment in uncemented implants according to Small et al. [9]. This correlation was not found in cemented implants, and no preoperative alignment was reported.

Although osteoarthritis (OA), the most common indication of TKA, was reported to be related to increased constitutional varus angles [43] and higher medial proximal preoperative BMD [44], no significant differences in density changes were found based on OA severity or TKA indication, respectively $[35,36]$.

In general, the findings over the studies indicate bone density to relatively shift towards the ML side which is 
increasingly loaded following postoperative knee alignment, relative to the preoperative situation. This change in ML density distribution is typically observed as an increase of bone loss in the load-decreased side but, in some studies, was (also) measured as densification in the predominantly loaded side (Table 2); however, a distribution shift was not observed over all alignment combinations in the studies, which could be influenced by implant-related factors.

\section{Preoperative Bone Density}

Few studies investigated the effect of preoperative BMD level on the course of periprosthetic bone density changes, with varying outcomes. Abu-Rajab et al. did not find a relationship between relative density changes and preoperative BMD [10]. Conversely, Li and Nilsson found that a higher preoperative BMD led to greater relative bone loss [11]. Hvid et al. concluded remodeling to be characterized by bone resorption in the denser condyle according to preoperative alignment, while BMD in the lower density condyle was constant over 2 years [34].

\section{Body Weight}

Higher body weight (BW) evidently leads to greater mechanical bone loading and, accordingly, has been linked to higher BMD measurements in non-TKA cohorts [45, 46]. Meanwhile, obesity has been associated with lower bone metabolism through different biochemical pathways [47], which could account for the lower rates of bone formation observed in obese postmenopausal women [48]. The same biochemical effects could play an important role in a twofold increased risk of revision surgery due to tibial aseptic loosening encountered in TKA patients with a BMI greater than $35 \mathrm{~kg} / \mathrm{m}^{2}$, regardless of knee alignment or age [49].

Several radiographic TKA studies failed to find a correlation between initial BMD values and BW or BMI [13, 34]. Interestingly, BW does seem to affect mid- to long-term bone density after TKA. Hvid et al. found a positive correlation between body weight index and BMD after 2 years [34]. Ishii et al. found that higher weight was associated with denser proximal tibiae more than 5 years after bilateral TKA [32], and higher lateral density in a study by Soininvaara et al. [35]. Small et al. only found this significant relation between BMI and relative density changes in cemented implants, but not in the uncemented fixation group [9].

\section{Activity Level}

The effect of subject activity on bone quality has been demonstrated in a number of bone density studies involving nonTKA population groups. Physical exercise had been found to positively affect general bone density in older adults and postmenopausal women, respectively [50,51]. Reduced activity, on the other hand, has been associated with bone loss, as observed by Leblanc et al. following 17 weeks of bed rest [52]. In turn, also quadricep muscle strength has been demonstrated to correlate with BMD in the proximal tibia in both in asymptomatic men and women, respectively $[53,54]$. However, similar effect of activity on BMD has not been demonstrated in bone density changes following TKA. Levels of activity and quadriceps strength have not been found to have a significant effect on periprosthetic BMD in different TKA studies $[32,34]$. The reason behind this deviating finding could be that effect of confined physiological loading is relatively small in comparison to effects of typical postoperative disuse and operation-induced bone loss, as put forward by Soininvaara et al. [35]. Bohr and Lundt concluded that reported postoperative BMD increase was the result of activity and weight-bearing due to improved function, based on the observation of a normal walking ability in all patients after 3 months, but no activity measures were used in this study [26].

\section{Discussion}

Implant stiffness and geometry were found to be implantrelated factors that have a significant impact on the course of tibial density over postoperative time. Greater implant stiffness leads to increased stress shielding, as higher material stiffness and increased baseplate thickness were both generally found to cause greater bone resorption. Geometry of the implant fixation features also affected density distributions, with a large cylindrical central stem displaying increased proximal and medial bone loss then multiple short pegs or a cruciform stem, respectively. No general differences in bone density were found due to fixation method, bearing type, or PCL retention.

Most of the reviewed patient-related factors were also not found to have a significant effect on postoperative density changes in the majority of relevant studies. Although age, sex, BW, and physical activity were found to affect tibial density in general non-TKA populations, their impact on the course of implant-induced remodeling was not demonstrated in most studies. Based on the consistency of conclusions in studies which did manage to find an effect of either of these factors, it can be assumed that increased age [9, 32], higher preoperative BMD [12], and lower subject BW [9, 32, 34, 35 ] at time of surgery were all associated with increased bone loss after TKA. These findings seem contradictory, since high preoperative BMD has been typically associated with younger [33] and heavier [45, 46] subjects, but this might indicate that different underlying factors play a role in related bone loss.

Knee alignment was the only patient-related factor which was consistently found to affect postoperative bone density 
distributions over multiple studies (Table 2). Postoperative varus alignment or correction towards varus (in case preoperative alignment was considered) typically led to relatively greater lateral bone loss, while valgus (corrected) implanted knees were associated with more relative bone loss on the medial side. This corresponds with computational remodeling outcome over a population of tibiae, in which increased medial bone loss is found systematically in neutral mechanical implant alignment over kinematic varus implant alignment simulations in constitutional varus native knees [55].

\section{Clinical Guideline Recommendations}

The clinical findings provide an overview of the effects of different factors on the course of tibial bone density over time. However, since each study used a unique study design, by using different combinations of baseline definitions, imaging techniques, ROIs, and follow-up time points, a direct comparison between reported data of different studies is not possible. In addition, several studies claiming to investigate the effect of a single implant-related factor were also found to vary other mechanical factors [14, 22, 23, 25]. There is also a large discrepancy between reported implantand subject-related measures over the studies; most studies do not report data on important patient- and implant-related factors which are out of the scope of their research question, such as knee alignment and implant stiffness, even though these factors have been demonstrated to influence the postoperative tibial density distribution $[14,21,22,35$, $36,40-42]$. Other factors known to influence general bone density, such as nutrition and medication, are not reported in any of the considered clinical densitometry studies.

In order to study the effect of a single TKA parameter on clinical bone remodeling, an in vivo experiment should be conducted with as little variation in other factors as possible. A clinical guideline could aid in considering and reporting of relevant implant-, surgery-, and patient-data and outcome measures, to prevent overlooked conflicting parameter variations in future studies. Another important benefit of such a general guideline is the potential to enable comparison over multiple studies between the effects of different mechanical factors, by defining additional consistent guidelines regarding study design, covering baseline measurements, follow-up time points, and ROI definitions, based on findings in current clinical studies.

\section{Baseline Measurements}

Various different baseline definitions have been used over the considered studies as a reference for tibial density changes over time. Most studies used the first postoperative density measure as the baseline value; the time point of this postoperative baseline measurement ranged from within the first 5 days [25] to as far as 1 year after operation [24]. Alternatively, other studies used preoperatively measured BMD as baseline [15, 21], or related bone changes to density in the unoperated contralateral knee at the same point in time $[10,18,19]$. All of these definitions are appropriate for studying bone density changes, but each has its characteristic advantages and disadvantages as explicated in Table 3.

Based on the advantages and disadvantages of each baseline method, we would recommend using a postoperative measurement within the first week after surgery, as a baseline for bone density measurements. Following this recommendation, effects of potential bone damage during surgery do not interfere with stress shielding-induced remodeling, and ROIs can be accurately reproduced based on the achieved implant position. A time span of 1 week from surgery is chosen as the recommended time frame for the baseline measurement to take place, to minimize remodeling effects before baseline, while ensuring it is clinically feasible to meet the recommendation for all subjects.

The number of included subjects ranged from 9 [26] to 309 [23] over the considered studies. We would recommend performing a power analysis before each study to determine the number of subjects needed to answer the research question, taking a possible loss to follow-up into account.

\section{Follow-up Time Points}

In line with the observations of baseline measurement time points, there is a large variation in follow-up time points found over the clinical studies. Total follow-up of the studies ranged from 1 year [35] to 10 years [9], with also a variety of different interval time points.

Single-photon emission CT (SPECT) uptake suggested implant-induced bone remodeling to only take place within the 2 two years after uncomplicated TKA, since the leveling of measured SPECT uptake in the second year indicated that bone metabolic activity was stable and a new equilibrium in bone turnover had been reached [56]. Since all sixteen subjects in this study did not show any clinical complications within the 2-year follow-up, cases with more extensive periprosthetic bone changes might take longer for this balance to be reached.

Ongoing bone density changes after 2 years were observed by other studies using DEXA [24, 40, 41], DRD [9, 22], and DPA [36]. These studies were consistent in their finding that most of the bone changes took place between the initial measurement intervals within the first 2 years; except for the studies of Petersen et al. and Levitz et al., which reported a large contribution of BMD changes at follow-up 
Table 3 Overview of advantages and disadvantages of the three baseline references found over the densitometry studies

\begin{tabular}{|c|c|c|}
\hline Baseline reference & Advantages & Disadvantages \\
\hline Contralateral tibia & $\begin{array}{l}\text { Normalization for age- and activity-related BMD } \\
\text { decline }\end{array}$ & $\begin{array}{l}\text { Only possible in unilateral TKA-contralateral TKA } \\
\text { during follow-up leads to costly exclusion } \\
\text { Assumption of tibial symmetry in terms of geometry } \\
\text { and density-more symmetrical knees are more } \\
\text { likely to receive bilateral TKA } \\
\text { Contralateral BMD changes due to progressing osteo- } \\
\text { arthritis skew implant-induced effects } \\
\text { Twice as much measurements-more time consuming } \\
\text { and accumulation of observational errors and radia- } \\
\text { tion exposure } \\
\text { Implant position not available as spatial reference for } \\
\text { ROI definition } \\
\text { Absolute BMD measurements are required-not } \\
\text { possible using cost-effective DRD }\end{array}$ \\
\hline Preoperative measurement & No additional clinical visits required & $\begin{array}{l}\text { Implant position not available as spatial reference for } \\
\text { ROI definition } \\
\text { Bone density outcome includes possible surgery- } \\
\text { related bone loss }\end{array}$ \\
\hline Initial postoperative measurement & Implant as spatial reference for ROI definition & $\begin{array}{l}\text { Baseline measurement might require additional visit } \\
\text { to clinic }\end{array}$ \\
\hline & $\begin{array}{l}\text { No effect of surgery-related bone damage on remod- } \\
\text { eling outcome }\end{array}$ & $\begin{array}{l}\text { Remodeling already in progress before baseline } \\
\text { measurement }\end{array}$ \\
\hline
\end{tabular}

in the third year and somewhere between 1 and 8 years, respectively [36, 41]. Ongoing long-term bone remodeling is likely to be caused more by systemic bone changes due to aging and physical activity over time, than by periprosthetic stress shielding, although no study was found including contralateral measurements over multiple follow-up time points. Based on the current studies, we would recommend to use a follow-up of at least 3 years and measure subjects after 3 , 6 , and 12 months, and each subsequent year from then on until follow-up, in addition to the recommended baseline measurement in the first week.

\section{Imaging Technique}

Tibial bone changes after TKA have been evaluated over the studies using four different imaging techniques. The most commonly used technique was DEXA, three studies have been found using DPA [26, 36, 41], two studies used DRD [9, 22], and two other studies made use of QCT [25, 34].

DEXA was concluded to be a reliable and precise standard for BMD measurements in the proximal tibia, with reproducibility errors between three consecutive scans, including repositioning, within $2.2 \%$ in any of three local regions [6]. DPA proved to be an alternative to DEXA in term of precision, with a coefficient of variation of $3 \%$ in the proximal tibia [57], but typically has lower spatial resolution, greater radiation exposure, and prolonged scan times compared to DEXA [58].
DRD is based on conventional radiographs and is, therefore, a cheaper alternative to DEXA. The technique was concluded to be a reliable replacement of DEXA in measuring relative density changes over time [8]. Disadvantages of the use of DRD are that it is not able to measure absolute BMD values, and the density normalization using intensity of the metal implant makes it not suitable for use in preoperative scans and all-poly implants.

QCT is the only three-dimensional imaging technique that is being used, enabling volumetric analysis of bone density. Reilly et al. concluded that QCT is reproducible in measuring BMD changes between different observers [7]. Downsides of QCT osteodensitometry are that it requires a higher radiation exposure than DEXA; measurements and analyses are more costly and time-consuming, and artifact reduction in case of metallic components introduces additional variability [59]. The use of QCT is therefore only recommended in cases where it is relevant to determine bone density changes at locations that cannot be measured using one or two DEXA projections, such as bone-specific cortical or trabecular regions.

It is important to be aware that measured absolute BMD values can vary between different imaging techniques, but also between different scanners using the same image modality [60], due to differences in hardware components, calibration, internal software processing, and measurement settings. We therefore recommend using a single system over all subjects and time points within a study. Due to the potential interference of scanner types, comparisons between 
different studies based on relative bone density changes over time are more reliable.

\section{Regions of Interest}

Each of the studies used different ROIs to study bone density in local areas of the proximal tibia. The number of ROIs ranged from just a single area [21-23, 32] to as much as 14 in QCT studies [25]. Definitions of ROIs differed greatly between studies. Most commonly, surface areas in anteroposterior (AP) projections were used, with one or more separate ROIs underneath the medial and lateral condyle, sometimes added with regions from ML projections. Different volumetric ROIs were used by studies based on QCT scans [25, 34], since this was the only three-dimensional imaging modality used.

Recommended ROIs should meet a few requirements to enable direct and reliable comparison between different studies, as a tibial alternative to the widely used Gruen zones in periprosthetic femoral hip remodeling [61]. Firstly, to enable consistent ROI definitions over all image modalities, the regions should be defined following a projected $2 \mathrm{D}$ view. This would require reconstruction from a fixed viewpoint in case of three-dimensional QCT imaging. Secondly, for the regional image intensities to accurately reflect associated bone densities, ROIs should not comprise bone cement pockets or metallic scattering following any of the modalities, meaning that the regional borders should not be defined too close to the implant interface. And, lastly, ROI locations should be accurately reproducible over all different tray designs, so they should not be defined based on the position of a central keel tip or fixation pegs. Given these requirements, it makes sense to define ROIs in the AP view of the proximal tibia. Regional medial and lateral densities following this viewpoint were found to be dependent on knee alignment according to multiple studies [11, 12, 35, 40], and the most clinical concern regarding tibial bone remodeling has been raised around medial tibial bone loss [3, 62].

Potential guideline ROIs have been used by the studies of Lonner et al. [19] and Minoda et al. [14], since they have applied consistent ROIs over multiple distinct implant designs. Lonner et al. had adopted the ROI definitions as used by Levitz et al. [36], which prescribed to use densities at coordinates medial and lateral directly underneath the baseplate, directly distal to the central keel tip, and $4 \mathrm{~cm}$ distal from the keel tip of the central stem implant. These four coordinates were positioned by Lonner et al. in such a way to not interfere with the fixation pegs or central stem in either of the two studied implant designs. Such ROI adjustment is not possible when comparing implants over multiple studies, and prescribed placement close to the baseplate and tip of a central keel makes it unsuitable to use in a clinical guideline due to the related dependency on implant design and possible implant scattering. The ROI definitions in the study of Minoda et al. are most suited to be recommended in a general clinical guideline, as ROI placement is irrespective of design features and at a distance from the implant baseplate (Fig. 2) [14]. Medial and lateral regions were defined $1 \mathrm{~cm}$ distal to the baseplate within the cortices of the proximal tibia, and the distal region was $4 \mathrm{~cm}$ distal from the center of the tibial baseplate. Each of the regions covered a square surface area of $1 \mathrm{~cm}^{2}$. Examination of all common primary tibial implant designs could determine whether this recommendation is feasible for a general guideline, or if it should be modified to enable use over more implants, by moving the two proximal ROIs more distally and reducing the surface area of the ROIs.

Using the implant position as spatial reference in defining ROIs is generally an accurate and reproducible way to locate bone regions, but it is important to be aware that excessive implant migration affects the included spatial bone volume and should be corrected for to compare consistent tibial regions over time.

\section{Data Reporting}

For an overview of the different mechanical factors playing a role in periprosthetic density changes, it would be strongly recommendable to report information on all implant-, subjectand surgery-related factors discussed in this review. Regarding each tibial tray used in the study, this includes the implant system and size, fixation type, stiffness (in terms of material,

\section{Regions of interest}

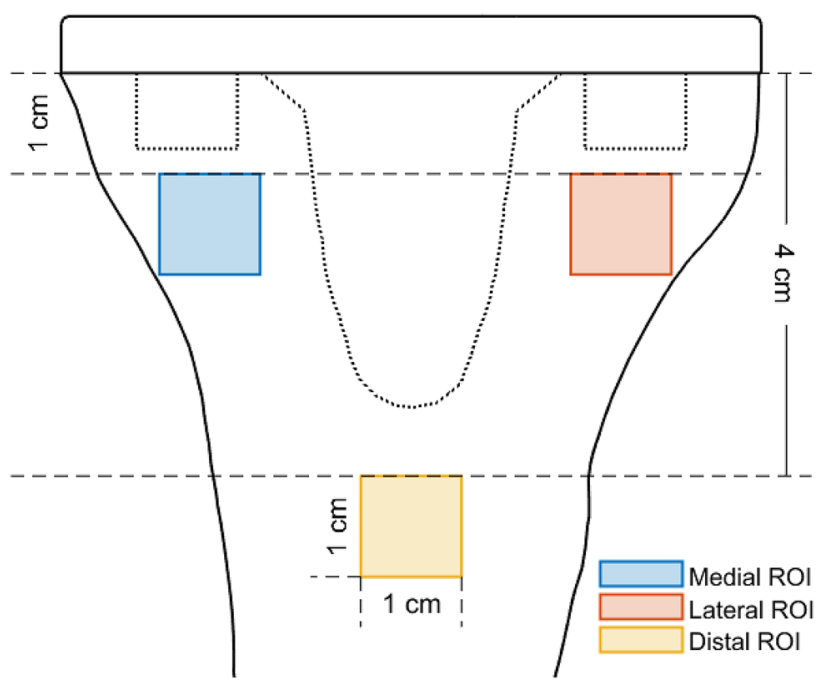

Fig. 2 Schematic AP view of ROI recommendations, following the definitions of Minoda et al. [14]. Further examination of current implant designs could lead to adjustment of the indicated reference distance measures, to make the recommended ROIs more generally applicable over different implants 
Table 4 Overview of clinical guideline recommendations

\begin{tabular}{|c|c|c|}
\hline Study design aspect & \multicolumn{2}{|l|}{ Recommendations } \\
\hline Baseline measurement & \multicolumn{2}{|c|}{$<1$ week postoperatively } \\
\hline Follow-up time points & \multicolumn{2}{|c|}{ 3, 6 months, 1, 2, 3 years postoperatively. Annual measurements in case of additional follow-up } \\
\hline Imaging technique & \multicolumn{2}{|c|}{$\begin{array}{l}\text { Use of DEXA preferred. QCT only in case of study-specific volumetric ROIs. DRD is an unfavored alternative when } \\
\text { no access to DEXA is possible }\end{array}$} \\
\hline Regions of interest & \multicolumn{2}{|c|}{$\begin{array}{l}3 \text { AP ROIs based on ROI definitions of Minoda et al. [14]: medial, lateral, and distal. Additional study-specific ROIs } \\
\text { optional }\end{array}$} \\
\hline \multirow[t]{4}{*}{ Data reporting } & Implant: & $\begin{array}{l}\text { Name, size, material, baseplate thickness, fixation features, } \\
\text { bearing type, PCL retention }\end{array}$ \\
\hline & Subject data: & $\begin{array}{l}\text { Age, sex, indication, BW, BMI, knee alignment pre- and } \\
\text { postoperatively, optional activity levels. Number of sub- } \\
\text { jects to be based on study-specific power analysis }\end{array}$ \\
\hline & Postoperative care: & $\begin{array}{l}\text { Prescribed medication, rehabilitation program, patient } \\
\text { instructions }\end{array}$ \\
\hline & Measurements: & $\begin{array}{l}\text { Imaging technique, scanner name and settings, ROI defini- } \\
\text { tions, absolute and relative BMD values at baseline, and } \\
\text { follow-up time points }\end{array}$ \\
\hline
\end{tabular}

baseplate thickness, and keel geometry), bearing type, and PCL retention. For subject data, this involves age, sex, condition, BW, BMI, pre- and postoperative knee alignments, and, if available, measured activity levels. Besides enabling a complete comparison between factors over different studies, considering all of these factors in the study design also makes researchers aware of any unintended (implant-related) factors that may interfere with answering a specific research question, as was found to be the case in different reported studies $[14,22,23,25]$. Clearly, not only the considered mechanical factors play a role in the course of postoperative bone remodeling, results between different patients and clinics may also largely differ due to varying success of surgical outcome, and differences in postoperative care, such as prescribed medication or rehabilitation program. It is therefore also advised to provide relevant information on the postoperative treatment plan and patient instructions.

Measurement results should be reported as completely as possible, including bone density values and optional activity measurements at all baseline and follow-up time points, in absolute and relative terms. Logically, it must be stated which imaging technique was used to obtain the bone densities, and sufficient details on how similar measurements could be reproduced, including name and relevant settings of the scanner, and accurate ROI definitions.

\section{Conclusions}

A general consensus on study design of future radiographic studies should enable reliable comparison between the effects of different TKA-related factors on postoperative bone density changes, which is currently not possible due to large variations in the design of available tibial densitometry studies. Based on reviewed studies, we proposed a guideline (Table 4), suggesting baseline and follow-up measurements using DEXA in the first postoperative week, and at 3 and 6 months and each consecutive year for at least 3 years, respectively, the use of three standardized ROIs, and adequate reporting of all relevant implant-, subject-, and surgery-related study data.

Author Contribution TA carried out the literature review and drafted the manuscript. IK participated in the design of the study, carried out the literature search, and helped to draft the manuscript. NV conceived of the study and participated in the design of the study. DJ participated in the design and coordination of the study and helped to draft the manuscript. All authors read and approved the final manuscript.

Funding Research grant by DePuy Synthes Joint Reconstruction, Leeds, UK.

The article does not contain any studies with human or animal subjects performed by any of the authors.

\section{Declarations}

Conflict of Interest The authors declare no competing interests.

Open Access This article is licensed under a Creative Commons Attribution 4.0 International License, which permits use, sharing, adaptation, distribution and reproduction in any medium or format, as long as you give appropriate credit to the original author(s) and the source, provide a link to the Creative Commons licence, and indicate if changes were made. The images or other third party material in this article are included in the article's Creative Commons licence, unless indicated otherwise in a credit line to the material. If material is not included in the article's Creative Commons licence and your intended use is not permitted by statutory regulation or exceeds the permitted use, you will need to obtain permission directly from the copyright holder. To view a copy of this licence, visit http://creativecommons.org/licenses/by/4.0/. 


\section{References}

1. Sharkey PF, Lichstein PM, Shen C, Tokarski AT, Parvizi J. Why are total knee arthroplasties failing today-has anything changed after 10 years? J Arthroplasty. 2013;29(9):1774-8.

2. Schroer WC, et al. Why are total knees failing today? Etiology of total knee revision in 2010 and 2011. J Arthroplasty. 2013;28(8 SUPPL):116-9.

3. Martin JR, Fehring KA, Watts CD, Springer BD, Fehring TK. Radiographic findings in patients with catastrophic varus collapse after total knee arthroplasty. J Arthroplasty. 2018;33(1):241-4.

4. Huiskes R, Weinans H, Grootenboer HJ, Dalstra M, Fudala B, Slooff TJ. Adaptive bone-remodeling theory applied to prostheticdesign analysis. J Biomech. 1987;20(11-12):1135-50.

5. Huiskes R, Weinans H, Van Rietbergen B. The relationship between stress shielding and bone resorption around total hip stems and the effects of flexible materials. Clin Orthop Relat Res. 1992;274:124-34.

6. Trevisan C, Bigoni M, Denti M, Marinoni EC, Ortolani S. Bone assessment after total knee arthroplasty by dual-energy X-ray absorptiometry: analysis protocol and reproducibility. Calcif Tissue Int. 1998;62(4):359-61.

7. Reilly K, Munro J, Pandit S, Kress A, Walker C, Pitto RP. Inter-observer validation study of quantitative CT-osteodensitometry in total knee arthroplasty. Arch Orthop Trauma Surg. 2007;127(8):729-31.

8. Hernandez-Vaquero D, Garcia-Sandoval MA, Fernandez-Carreira JM, Suarez-Vázquez A, Perez-Hernández D. Measurement of bone mineral density is possible with standard radiographs: a study involving total knee replacement. Acta Orthop. 2005;76(6):791-5.

9. Small SR, Ritter MA, Merchun JG, Davis KE, Rogge RD. Changes in tibial bone density measured from standard radiographs in cemented and uncemented total knee replacements after ten years' follow-up. Bone Jt J. 2013;95(7):911-6.

10. Abu-Rajab RB, Watson WS, Walker B, Roberts J, Gallacher SJ, Meek RMD. Peri-prosthetic bone mineral density after total knee arthroplasty. J Bone Jt Surg - Ser B. 2006;88(5):606-13.

11. Li MG, Nilsson KG. Changes in bone mineral density at the proximal tibia after total knee arthroplasty: a 2-year follow-up of 28 knees using dual energy X-ray absorptiometry. J Orthop Res. 2000;18(1):40-7.

12. Li MG, Nilsson KG. The effect of the preoperative bone quality on the fixation of the tibial component in total knee arthroplasty. J Arthroplast. 2000;15(6):744-53.

13. Li MG, Nilsson KG. No relationship between postoperative changes in bone density at the proximal tibia and the migration of the tibial component 2 years after total knee arthroplasty. J Arthroplast. 2001;16(7):893-900.

14. Minoda $\mathrm{Y}$, Kobayashi A, Ikebuchi M, Iwaki H, Inori F, Nakamura H. Porous tantalum tibial component prevents periprosthetic loss of bone mineral density after total knee arthroplasty for five yearsa matched cohort study. J Arthroplast. 2013;28(10):1760-4.

15. Winther N, Jensen C, Petersen M, Lind T, Schrøder H, Petersen $\mathrm{M}$. Changes in bone mineral density of the proximal tibia after uncemented total knee arthroplasty. A prospective randomized study. Int Orthop. 2016;40(2):285-94.

16. Petersen MM, Gehrchen PM, Østgaard SE, Nielsen PK, Lund B. Effect of hydroxyapatite-coated tibial components on changes in bone mineral density of the proximal tibia after uncemented total knee arthroplasty: a prospective randomized study using dualenergy x-ray absorptiometry. J Arthroplast. 2005;20(4):516-20.

17. Choi K, Kuhn JL, Ciarelli MJ, Goldstein SA. The elastic moduli of human subchondral, trabecular, and cortical bone tissue and the size-dependency of cortical bone modulus. J Biomech. 1990;23(11):1103-13.

18. Harrison AK, Gioe TJ, Simonelli C, Tatman PJ, Schoeller MC. Do porous tantalum implants help preserve bone?: evaluation of tibial bone density surrounding tantalum tibial implants in TKA. Clin Orthop Relat Res. 2010;468(10):2739-45.

19. Lonner JH, Klotz M, Levitz C, Lotke PA. Changes in bone density after cemented total knee arthroplasty: influence of stem design. J Arthroplast. 2001;16(1):107-11.

20. Houdek MT, Wagner ER, Wyles CC, Watts CD, Cass JR, Trousdale RT. all-polyethylene tibial components: an analysis of long-term outcomes and infection. J Arthroplast. 2016;31(7):1476-82.

21. Martin JR, Watts CD, Levy DL, Miner TM, Springer BD, Kim $\mathrm{RH}$. Tibial tray thickness significantly increases medial tibial bone resorption in cobalt-chromium total knee arthroplasty implants. J Arthroplast. 2017;32(1):79-82.

22. Wong DW, Lee QJ, Lo CK, Wong YC, Law KW, Li AH. Tibial tray thickness does not significantly increase medial tibial bone resorption: Using tibial bone density as an objective measurement method. Knee. 2020;27(2):572-9.

23. Yoon C, Chang MJ, Chang CB, Song MK, Shin JH, Kang SB. Medial tibial periprosthetic bone resorption and its effect on clinical outcomes after total knee arthroplasty: cobalt-chromium vs titanium implants. J Arthroplast. 2018;33(9):2835-42.

24. Hernandez-Vaquero D, Garcia-Sandoval MA, Fernandez-Carreira JM, Gava R. Influence of the tibial stem design on bone density after cemented total knee arthroplasty: a prospective seven-year follow-up study. Int Orthop. 2008;32(1):47-51.

25. Munro JT, Pandit S, Walker CG, Clatworthy M, Pitto RP. Loss of tibial bone density in patients with rotating- or fixed-platform TKA. Clin Orthop Relat Res. 2010;468(3):775-81.

26. Bohr $\mathrm{HH}$, Lund $\mathrm{B}$. Bone mineral density of the proximal tibia following uncemented arthroplasty. J Arthroplast. 1987;2(4):309-12.

27. Robertsson O, Sundberg M, Sezgin EA, Lidgren L, W-Dahl A. Higher risk of loosening for a four-pegged tka tibial baseplate than for a stemmed one: a register-based study. Clin Orthop Relat Res. 2020;478(1):58-65.

28. Bertin KC. Tibial component fixation in total knee arthroplasty a comparison of pegged and stemmed designs. J Arthroplast. 2007;22(5):670-8.

29. Miller CW, Pettygrow R. Long-term clinical and radiographic results of a pegged tibial baseplate in primary total knee arthroplasty. J Arthroplast. 2001;16(1):70-5.

30. Tjørnild M, Søballe K, Hansen PM, Holm C, Stilling M. Mobilevs. Fixed-bearing total knee replacement: a randomized radiostereometric and bone mineral density study. Acta Orthop. 2015;86(2):208-14.

31. Saari T, Uvehammer J, Carlsson L, Regnér L, Kärrholm J. Joint area constraint had no influence on bone loss in proximal tibia 5 years total knee replacement. J Orthop Res. 2007;25(6):798-803.

32. Ishii Y, Noguchi H, Sato J, Ishii H, Todoroki K, Toyabe SI. Association between body weight and proximal tibial bone mineral density after bilateral total knee arthroplasty. Knee. 2017;24(5):1153-9.

33. Runolfsdottir HL, Sigurdsson G, Franzson L, Indridason OS. Gender comparison of factors associated with age-related differences in bone mineral density. Arch Osteoporos. 2015;10(1):214.

34. Hvid I, Bentzen SM, Jørgensen J. Remodeling of the tibial plateau after knee replacement: CT bone densitometry. Acta Orthop. 1988;59(5):567-73.

35. Soininvaara TA, Miettinen HJA, Jurvelin JS, Suomalainen OT, Alhava EM, Kröger HPJ. Periprosthetic tibial bone mineral density changes after total knee arthroplasty: one-year follow-up study of 69 patients. Acta Orthop Scand. 2004;75(5):600-5.

36. Levitz CL, Lotke PA, Karp JS. Long-term changes in bone mineral density following total knee replacement. Clin Orthop Relat Res. 1995;(321):68-72.

37. Bellemans J, Colyn W, Vandenneucker H, Victor J. The chitranjan ranawat award. Clin Orthop Relat Res. 2012;470(1):45-53. 
38. Hulet C, Sabatier JP, Souquet D, Locker B, Marcelli C, Vielpeau C. Distribution of bone mineral density at the proximal tibia in knee osteoarthritis. Calcif Tissue Int. 2002;71(4):315-22.

39. Thorp LE, et al. Bone mineral density in the proximal tibia varies as a function of static alignment and knee adduction angular momentum in individuals with medial knee osteoarthritis. Bone. 2006;39(5):1116-22.

40. Jaroma A, Soininvaara T, Kröger H. Periprosthetic tibial bone mineral density changes after total knee arthroplasty: a 7-year follow-up of 86 patients. Acta Orthop. 2016;87(3):268-73.

41. Petersen MM, Nielsen PT, Lauritzen JB, Lund B. Changes in bone mineral density of the proximal tibia after uncemented total knee arthroplasty: a 3-year follow-up of 25 knees. Acta Orthop. 1995;66(6):513-6.

42. Shibuki T, Sukezaki F, Suzuki T, Toyoshima Y, Nagai T, Inagaki K. Periprosthetic bone mineral density changes after cementless total knee arthroplasty. Showa Univ J Med Sci. 2016;28(2):155-61.

43. Victor JMK, Bassens D, Bellemans J, Gürsu S, Dhollander AAM, Verdonk PCM. Constitutional varus does not affect joint line orientation in the coronal plane knee. Clin Orthop Relat Res. 2014;472(1):98-104.

44. Karvonen RL, Miller PR, Nelson DA, Granda JL, FernandezMadrid F. Periarticular osteoporosis in osteoarthritis of the knee. J Rheumatol. 1998;25(11):2187-94.

45. Blain H, Vuillemin A, Teissier A, Hanesse B, Guillemin F, Jeandel $C$. Influence of muscle strength and body weight and composition on regional bone mineral density in healthy women aged 60 years and over. Gerontology. 2001;47(4):207-12.

46. Yang $\mathrm{S}$, Shen X. Association and relative importance of multiple obesity measures with bone mineral density: the National Health and Nutrition Examination Survey 2005-2006. Arch Osteoporos. 2015;10(1):1-8.

47. Savvidis C, Tournis S, Dede AD. Obesity and bone metabolism. Hormones. 2018;17(2):205-17.

48. Papakitsou EF, et al. Body mass index (BMI) and parameters of bone formation and resorption in postmenopausal women. Maturitas. 2004;47(3):185-93.

49. Abdel MP, Bonadurer GF III, Jennings MT, Hanssen AD. Increased aseptic tibial failures in patients with a BMI $\geq 35$ and well-aligned total knee arthroplasties. J Arthroplast. 2015;30(12):2181-4.

50. Marques EA, Mota J, Carvalho J. Exercise effects on bone mineral density in older adults: a meta-analysis of randomized controlled trials. Age (Omaha). 2012;34(6):1493-515.

51. Kelley GA, Kelley KS, Kohrt WM. Effects of ground and joint reaction force exercise on lumbar spine and femoral neck bone mineral density in postmenopausal women: a meta-analysis of randomized controlled trials. BMC Musculoskelet Disord. 2012;13:177.

52. Leblanc AD, Schneider VS, Evans HJ, Engelbretson DA, Krebs JM. Bone mineral loss and recovery after 17 weeks of bed rest. J Bone Miner Res. 1990;5(8):843-50.

53. Pettersson U, Nordström P, Lorentzon R. A comparison of bone mineral density and muscle strength in young male adults with different exercise level. Calcif Tissue Int. 1999;64(6):490-8.

54. Madsen OR, Schaadt O, Bliddal H, Egsmose C, Sylvest J. Relationship between quadriceps strength and bone mineral density of the proximal tibia and distal forearm in women. J Bone Miner Res. 1993;8(12):1439-44.

55. Anijs T, Wolfson T, Verdonschot N, Janssen D. Population-based effect of total knee arthroplasty alignment on simulated tibial bone remodeling. J Mech Behav Biomed Mater. 2020.

56. Soininvaara T, Nikola T, Vanninen E, Miettinen H, Kröger H. Bone mineral density and single photon emission computed tomography changes after total knee arthroplasty: a 2-year follow-up study. Clin Physiol Funct Imaging. 2008;28(2):101-6.

57. Bohr HH, Schaadt O. Mineral content of upper tibia assessed by dual photon densitometry. Acta Orthop. 1987;58(5):557-9.

58. Mazess RB, Barden HS. Measurement of bone by dual-photon absorptiometry (DPA) and dual-energy X-ray absorptiometry (DEXA). Ann Chir Gynaecol. 1988;77(5-6):197-203.

59. Schmidt R, et al. Inter- and intraobserver assessment of periacetabular osteodensitometry after cemented and uncemented total hip arthroplasty using computed tomography. Arch Orthop Trauma Surg. 2005;125(5):291-7.

60. Free J, et al. The effect of different CT scanners, scan parameters and scanning setup on Hounsfield units and calibrated bone density: a phantom study. Biomed Phys Eng Express. 2018;4(5).

61. Gruen TA, McNeice GM, Amstutz HC. 'Modes of failure' of cemented stem-type femoral components: a radiographic analysis of loosening. Clin Orthop Relat Res. 1979;(141):17-27.

62. Fehring TK, Fehring KA, Anderson LA, Otero JE, Springer BD. Catastrophic varus collapse of the tibia in obese total knee arthroplasty. J Arthroplast. 2017;32(5):1625-9.

Publisher's Note Springer Nature remains neutral with regard to jurisdictional claims in published maps and institutional affiliations. 